\title{
Investigación experimental en el consumo de energía específica y reducción de tamaño en la molienda de bolas usando diferentes tipos de levantador

An experimental study of specific energy consumption and size reduction in ball grinding
mill using different types of lifter

\section{Rosales-Marín Gilberto \\ Universidad Autónoma de San Luis Potosí \\ Coordinación Académica Región Altiplano \\ Correo: gilberto.rosales@uaslp.mx \\ https://orcid.org/0000-0003-3679-3981}

Andrade-Martínez Jonatan

Universidad Autónoma de San Luis Potosí

Coordinación Académica Región Altiplano

Correo: jonatan.andrade@uaslp.mx

https://orcid.org/0000-0003-2899-031X

Alvarado-Macías Gabriela

Universidad Autónoma de San Luis Potosí

Coordinación Académica Región Altiplano

Correo.gabriela.alvarado@uaslp.mx

https://orcid.org/0000-0001-8504-2485

\author{
Espinosa-Serrano Erik \\ Universidad Autónoma de San Luis Potosí \\ Coordinación Académica Región Altiplano \\ Correo: erik.espinosa@uaslp.mx \\ https://orcid.org/0000-0001-7591-9140 \\ Nava-Gómez Dora \\ Universidad Autónoma de San Luis Potosí \\ Coordinación Académica Región Altiplano \\ Correo:dora.nava@uaslp.mx \\ https://orcid.org/0000-0002-2651-6246
}

\section{Resumen}

En este artículo una muestra mineral polimetálico con contenidos en ley de cobre (0.65 \%), plomo (1.3 \%) y zinc (2.3\%) se somete a reducción de tamaño usando un molino de bolas. Se diseñaron cuatro tipos de levantadores con variaciones en altura (h) y el ángulo $(\alpha)$. Cinéticas de molienda se realizaron con tiempos de 5, 10 y 15 min con cada tipo de levantador. Una prueba adicional se ha realizado sin considerar ningún tipo de levantador. El estudio arroja que en general los levantadores magnifican la molienda, ya que estos generan las corrientes de catarata y cascada que dan origen a los mecanismos de molienda de impacto y abrasión. El análisis de la cantidad de finos obtenidos contra la energía específica demuestra que sin ningún tipo de levantador la molienda se da por el mecanismo de abrasión, el cual genera fractura del mineral lenta. Las cinéticas de molienda cuando hubo uso de levantadores denotan que el Levantador IV $\left(\alpha=22.5^{\circ} ; \mathrm{h}=12 \mathrm{~mm}\right)$ tiene la mejor eficiencia en la molienda de bolas, ya que tiene un consumo de energía de $9.69 \mathrm{kWh} /$ ton y produce la mayor cantidad de finos (32 \%) de los levantadores propuestos. Tanto la altura, así como el ángulo del levantador, son parámetros que deben considerarse para futuros diseños de este tipo de aditamentos internos de la molienda de bolas, ya que su efecto es significativo.

Descriptores: Molino de bolas, geometría de levantadores, reducción de tamaño, procesamientos de minerales, perfil de carga.

\begin{abstract}
In this paper, a polymetallic mineral sample with a grade of copper (0.65\%), lead (1.3\%) and zinc (2.3\%) is subjected to size reduction using a ball mill. Four types of lifters were designed with variations in height $(h)$ and angle $(\alpha)$. Grinding kinetics were performed using times of 5, 10 and 15 min with each type of lifter. An additional test has been performed without considering any type of lifter. The study shows that in general, the lifters magnify the grinding, due, the lifters generate the cataract and waterfall streams, this type of streams generate the impact and abrasion grinding mechanisms. The analysis of the number of fines obtained against the specific energy, demonstrates that without any type of lifter the grinding is given by the abrasion mechanism, which generates a slow mineral fracture. The grinding kinetics using lifters denote that Lifter IV ( $\left.\alpha=22.5^{\circ} ; \mathrm{h}=12 \mathrm{~mm}\right)$ has the best efficiency in grinding balls, due, the energy consumption of $9.69 \mathrm{kWh} /$ ton and produces the highest number of fines $(32 \%)$ of the proposed lifters. The height and the angle of the lifter are parameters that should be considered for future designs of this type of internal grinding ball attachments, due the produced effect is significant.
\end{abstract}

Keywords: Ball mill, lifter geometry, size reduction, mineral processing, charge profile. 


\section{INTRODUCCIÓN}

En la actualidad existen distintos procesos unitarios que deben llevarse a cabo para obtener los minerales de valor comercial y de los cuales se obtenga un beneficio económico. Dentro de estos procesos unitarios, es la molienda, la que genera un incremento en los costos de operación debido al consumo de energía y metal. La principal razón de someter los minerales a un proceso de reducción de tamaño es liberar los elementos valiosos de la matriz generalmente integrada por silicatos, carbonatos o arcillas. Las operaciones de reducción de tamaño conllevan transformaciones físicas que son indispensables para los posteriores procesos de concentración (Colman, 1983).

Los levantadores son aditamentos empleados para aumentar la vida útil de los revestimientos del molino y mejorar la eficiencia de molienda. Los levantadores tienen como función principal el transferir la energía rotacional del molino hacia la carga influyendo así en el rendimiento de fractura, en consecuencia, en el rendimiento del equipo. Sin embargo, debido a colisiones de bolas y partículas con revestimientos en molinos, los revestimientos se desgastan y cambian de perfil. El rendimiento de la molienda cambiará correspondientemente a lo largo de la vida útil de los revestimientos. Por lo tanto, conocer el efecto de los perfiles del levantador y el cómo evoluciona su desgaste contribuirá entre otras cosas a: Elegir el mejor diseño de levantador que optimice el rendimiento del molino durante su vida útil, determinar el tiempo óptimo de reemplazo del levantador y reducir los consumos de energía logrando la máxima reducción posible. Varios investigadores han hecho aportaciones para estudiar los molinos de bolas a lo largo de dos décadas (Rajamani \& Mishra, 1996; Morrison \& Cleary, 2008; Djordjevic, 2005; Powell \& Mc Bride, 2006; Geng et al., 2015; Bbosa et al., 2016) por mencionar algunos. Rajamani \& Mishra (1996) explicaron la influencia de la velocidad en el rompimiento de partículas usando un molino de acrílico en dos dimensiones. Cleary (1998) estudió en gran detalle el efecto de la velocidad en el consumo de energía, para esto, el torque y la potencia fueron medidas.

El estudio cinético para fracturar minerales se ha empleado en distintos estudios para entender la interacción de los mecanismos que llevan a cabo la fractura de mineral. Rosales et al. (2016) estudiaron la fractura por compresión en lecho confinado. Los procesos de molienda de bolas en húmedo es una metodología novedosa, ya que desde el punto de vista industrial, las cinéticas de molienda en húmedo arrojan mejores aproximaciones que la molienda en seca (Bhattachary- ya, 2016). En el presente trabajo cinéticas de molienda húmedas bajo condiciones operacionales usadas a nivel industrial son usadas para medir el efecto de distintos tipos de levantadores.

\section{ANTECEDENTES TEÓRICOS}

\section{LA MOLIENDA MECÁNICA EN LOS MOLINOS DE BOLAS}

La molienda mecánica (MM), también conocida como fractura mecánica, es un proceso para reducir el tamaño físico de las partículas sólidas gruesas a su nivel microestructural. Este tipo de molienda se usa con frecuencia para reducir el tamaño de las partículas en la metalurgia de polvos, cerámica y minería. En la molienda mecánica, se utilizan diferentes medios de molienda que van desde barras, bolas de acero, alúmina y guijarros de circonio, por mencionar algunos.

Cuando comienza un proceso de molienda, el movimiento de los materiales de carga (bolas, minerales y agua) da lugar a muchas zonas que se rigen por diferentes mecanismos de fractura. Moys et al. (1996) muestran el comportamiento típico de la carga del molino con la posición de la carga de medios como se muestra en la Figura 1. En el proceso de molienda se pueden distinguir dos mecanismos de fractura principales, colisión y fricción. Se entiende que la colisión da origen a impacto, generalmente este mecanismo de molienda es el resultado de la corriente de catarata, en este punto, el medio de molienda (bolas) tiene una trayectoria parabólica. Este tipo de mecanismo produce una alta rotura con poca energía. Por otro lado, la fricción da origen a la abrasión. Este mecanismo de fractura generalmente genera un movimiento en cascada con una trayectoria de bola circular y produce una buena cantidad de partículas finas, desafortunadamente, la ruptura de partí-

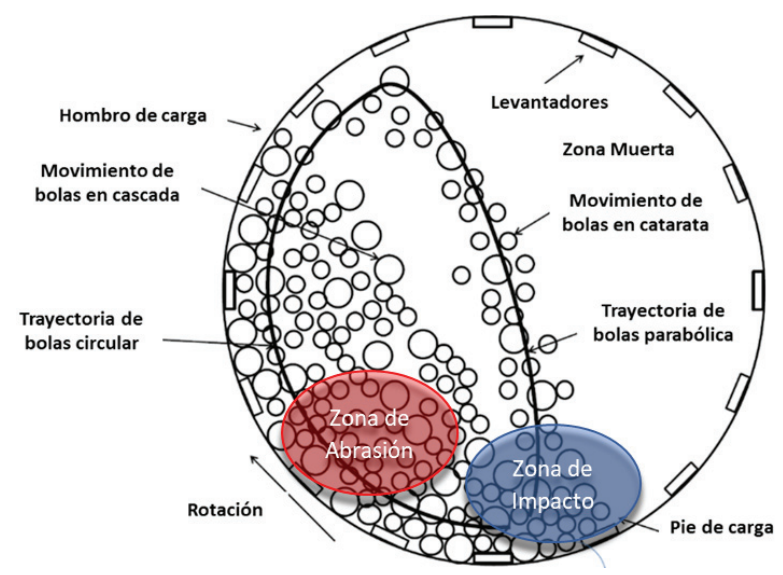

Figura 1. Perfil de carga en el molino de bolas en un evento de molienda 
culas finas requiere una gran cantidad de tiempo y energía. Esto se debe a que la acción de fricción crea abrasión generando calor en el sistema, esta es una de las principales pérdidas de energía en este mecanismo de molienda.

Powell \& McBride (2004) definieron la carga del hombro como el punto más alto en el que el material sale de la carcasa del molino, en este punto es donde comienza la corriente de catarata. El pie de carga es el punto donde ocurre la intersección del movimiento de volteo de la carga con la corriente de catarata. Esta es la zona donde el impacto es el mecanismo predominante de reducción de tamaño. La zona donde se da la molienda por abrasión se localiza donde se da el movimiento de cascada hasta antes del pie de carga. La evaluación de la geometría de los levantadores propuestos se basa en tratar de equilibrar estos mecanismos, preferentemente el de impacto, debido a que este mecanismo resulta en un alto índice de fractura.

\section{CÁlCUlo de la eNerGía en UN MOLINO DE BOLAS}

Por definición el índice de trabajo Wi corresponde a los $\mathrm{kWh}$ /ton requeridos para moler una mena desde un tamaño de alimentación infinito hasta un producto de $100 \mu \mathrm{m}$ (Bond, 1961). La energía requerida (E) para un evento de molienda es proporcional a la energía suministrada, para su cálculo debe considerarse el voltaje $(V)$, el amperaje $(A)$ y las fases del motor $(F)$. La expresión para el cálculo de dicha energía se muestra a continuación:

$$
E=\frac{V \cdot A \cdot \sqrt{F}}{1000}
$$

La potencia nominal consumida $(P)$ puede calcularse también como se muestra en la Ecuación 2. En esta relación el consumo de energía en un molino está relacionado con el torque:

$$
P=\frac{2 \pi N \tau}{60}
$$

Donde:

$N=$ velocidad del molino en $\mathrm{rpm}$

$\tau=$ torque ejercido por el molino considerando la fricción en los rodamientos

Considerando la energía adicionada al sistema $(P)$, el tiempo total de molienda $\left(t_{c}\right)$ en minutos y la cantidad de muestra $(m)$ en kilogramos. La energía específica (Ee) puede calcularse como:

$$
E e=\frac{P t_{c}}{m}
$$

\section{TRABAJO EXPERIMENTAL}

\section{TIPOS DE LEVANTADORES PROPUESTOS}

En el procesamiento de minerales se han utilizado diferentes geometrías de levantadores en las que se incluyen tipo trapezoidal, cuadrada, ondulada y circunferencial reversible. Cada una de estas geometrías se diseñaron para reducir los consumos de energía, mientras favorecen un patrón de movimiento de carga óptimo para ayudar a moler eficientemente, así como para reducir los requisitos de mantenimiento. Para esta parte de la experimentación se usaron 8 levantadores de acero al carbón con el mismo espacio entre ellos. La geometría del Levantador I, es del tipo cuadrado, mientras que las otras tres geometrías (Tipo II, Tipo III, Tipo IV) muestran una variación en las caras de sus ángulos y en sus alturas. La densidad específica del acero al carbón es de $7.84 \mathrm{~g} / \mathrm{cm}^{3}$ (Figura 2).

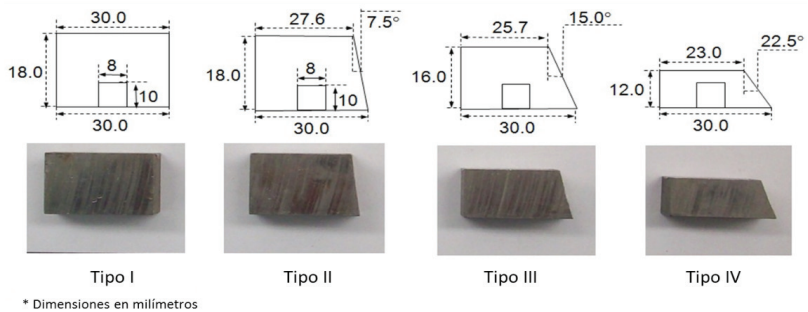

Figura 2. Geometría de levantador que imita desgaste

\section{Cinéticas de mOlienda (MOlino de BOlas)}

Una muestra de $8.3 \mathrm{~kg}$ de mineral polimetálico con contenidos en ley de cobre $(0.65 \%)$, plomo $(1.3 \%)$ y zinc $(2.3 \%)$ cuya densidad es de $3.16 \mathrm{~g} / \mathrm{cm}^{3}$ fue homogenizada y tamizada; los tamaños de partícula determinados fueron entre 6,300 y $38 \mu \mathrm{m}$. El molino de laboratorio empleado para las pruebas en lote tiene un diámetro interno de $0.32 \mathrm{~m}$ y una longitud de $0.35 \mathrm{~m}$ (Figura 3 ). El nivel de carga fue de $40 \%$ en todas las pruebas. Para variar la velocidad del molino, en el motor se instaló una unidad de control digital. La velocidad usada fue de $48.59 \mathrm{rpm}$ ( $65 \%$ de $V c$ ) a un porcentaje de sólidos de $60 \%$, el tamaño óptimo de bola fue calculado usando la ecuación (4) (Bond, 1961). 


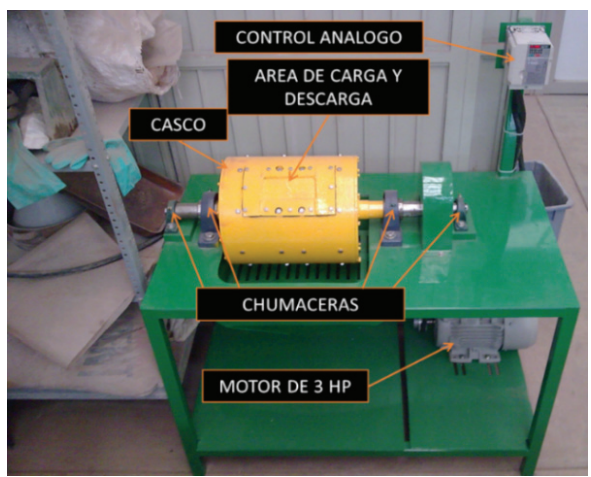

Figura 3. Molino experimental

$B_{S}=1.354\left(F_{80}\right)^{0.5}\left[\frac{\rho_{\text {ore }} W_{i B}}{N_{c} D^{0.5}}\right]^{\frac{1}{3}}$

Donde:

$B_{S}=$ tamaño óptimo de bola

$F_{80}=$ a $80 \%$ de la fracción pasante en la alimentación fresca

$D$ = diámetro interno del molino

$\rho_{o}=$ gravedad específica del mineral

$W_{i b}$ fue calculado en 6 ciclos siguiendo la metodología experimental de Bond (1961). El tamaño del collar de

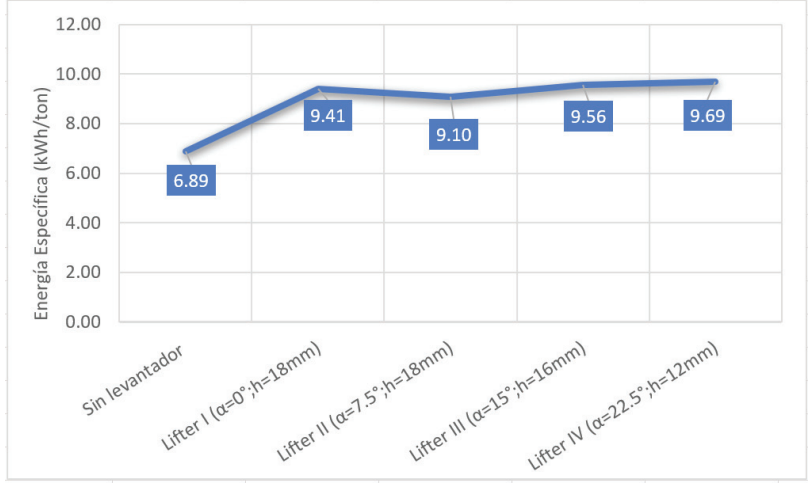

bolas $W_{i b}$ dentro del molino fue calculado acorde con la distribución de Gates-Gaudin-Schumann:

$G(x)=100\left[\frac{B_{s}}{k_{100}}\right]^{m}$

Donde:

$G(x)=$ porcentaje retenido en la malla " $x$ "

$k_{100}=$ a $100 \%$ pasante en la función lineal

$m$ = pendiente

El tamaño de distribución de las bolas se muestra en la Tabla 1.

\section{RESULTADOS Y DISCUSIONES}

Comparación de CONSUMOS DE ENERGÍA ESPECífica $(\mathrm{KWH} / \mathrm{TON})$

En las pruebas cinéticas de molienda se midió la cantidad de energía suministrada usando un medidor analógico. Este medidor analógico es capaz de tomar cerca de 125 mediciones por minuto. El voltaje y el amperaje fueron transformados a $\mathrm{kWh}$ y expresado en términos de energía específica (kWh/ton) usando la Ecuación 3, la cual representa la cantidad kilowatt por hora suministrados para obtener una tonelada de mineral.

La Figura 4 muestra el consumo de energía específica bajo condiciones levantadores propuestos (I, II, III y

Figura 4. Consumo de potencia con los diferentes tipos de levantador

Tabla 1. Parámetros del modelo $G(x)$ y collar de bolas usado

\begin{tabular}{|c|c|c|c|c|c|}
\hline \multicolumn{4}{|c|}{ Características Experimentales } & \multirow[t]{2}{*}{$\begin{array}{l}\text { Tamaño de bola } \\
(\mathrm{mm}) \mathrm{m}=4.8\end{array}$} & \multirow[t]{2}{*}{$\begin{array}{c}\text { Distribución } \\
\text { k100 }=76.2\end{array}$} \\
\hline $\mathrm{Wi}(\mathrm{kWh} /$ ton $)$ & 22.6 & Mena & Polimetálica & & \\
\hline Potencia Motor (Hp) & 3 & $\rho$ Ore $\left(\right.$ ton $\left./ \mathrm{m}^{3}\right)$ & 3.16 & 76.2 & 48.7 \\
\hline $\mathrm{D}(\mathrm{m})$ & 0.32 & $\mathrm{~F} 80(\mu)$ & 9500 & 63.5 & 49.6 \\
\hline Largo (m) & 0.35 & Velocidad crítica (\%) & 65 & 50.8 & 1.65 \\
\hline Nivel de Carga (\%) & 40 & Tiempo de molienda (min) & 5,10 y 15 & & \\
\hline
\end{tabular}


IV) adicionalmente se realizó una prueba con distribución granulométrica sin el uso de ningún levantador, esto para poder identificar el efecto en cuestión de reducción de tamaño en cada levantador. Como es de esperarse, la muestra sometida a molienda sin el uso de un levantador tiene un consumo de energía de $6.89 \mathrm{kWh} /$ ton es el consumo más bajo registrado en esta serie de pruebas, por otro lado, el consumo de energía más elevado lo tiene el Levantador IV el cual tiene un ángulo $\alpha=22.5^{\circ} \mathrm{y}$ tiene $12 \mathrm{~mm}$ de altura. Claramente en la Figura 5 podemos deducir que existe una correlación inversamente proporcional entre la altura del levantador y el consumo de energía, ya que a medida que la altura decrece el consumo de energía claramente se incrementa. La prueba que tiene el menor consumo de energía cuando se usa un levantador es la que corresponde al Levantador II cuyo ángulo $\alpha=7.5^{\circ}$. Al considerar las muestras sometidas a molienda con levantadores que tienen un ángulo (Levantadores II, III y IV), la tendencia de estas pruebas denota que, ante el incremento del ángulo, el consumo de energía aumenta.

\section{ANÁLISIS DEL PRODUCTO GRANULOMÉTRICO DE MOLIENDA CON LEVANTADORES PROPUESTOS}

A continuación (Figura 5) se muestran los análisis granulométricos obtenidos con cada uno de los levantadores propuestos, considerando cinéticas de molienda de 5, 10 y $15 \mathrm{~min}$.

En la Figura 5 se ha graficado la granulometría de alimentación, que ha sido la misma para cada muestra molida con diferente levantador. Como se puede apreciar la cinética con el Levantador I $\left(\alpha=0^{\circ} ; \mathrm{h}=18 \mathrm{~mm}\right)$ cuya configuración es cuadrada, tiene una cinética lenta, esto puede deducirse ya que tomando como referencia la línea de alimentación cada uno de los tiempos de molienda (5, 10 y 15 min) están muy cercanos a esta. Bajo este mismo concepto de referencia la cinética de molienda más rápida es aquella que tiene el Levantador IV $\left(\alpha=22.5^{\circ} ; \mathrm{h}=12 \mathrm{~mm}\right)$ ya que cualquiera de los tiempos de molienda realizados con este tipo de levantador se aleja considerablemente de la línea de alimentación, con esto, se puede deducir que el Levantador IV es el que es más propicio para acelerar la molienda en el molino de bolas, donde los primeros $5 \mathrm{~min}$ son clave para realizar esta reducción, ya que como se aprecia en el gráfico a este tiempo se consigue una fractura más acelerada si se compara con los otros levantadores usados en este estudio. La cinética de molienda sin ningún tipo de levantador fue analizada, sin embargo, como se puede apreciar en la Figura 6, independientemente del tiempo transcurrido prácticamente la reducción de tamaño no se puede llevar acabo. Es decir, el uso de cualquier tipo de levantador incrementa significativamente la molienda.

La Figura 7 muestra las gráficas utilizando un tamaño adimensional que se normaliza por el tamaño medio, $X_{50}$. Este tamaño, se define como el porcentaje de partículas que tiene la misma posibilidad de ir a la fracción gruesa como a la fracción fina (Hosten \& Cimilli, 2009; Bulled et al., 2008). El número adimensional $X / X_{50}$
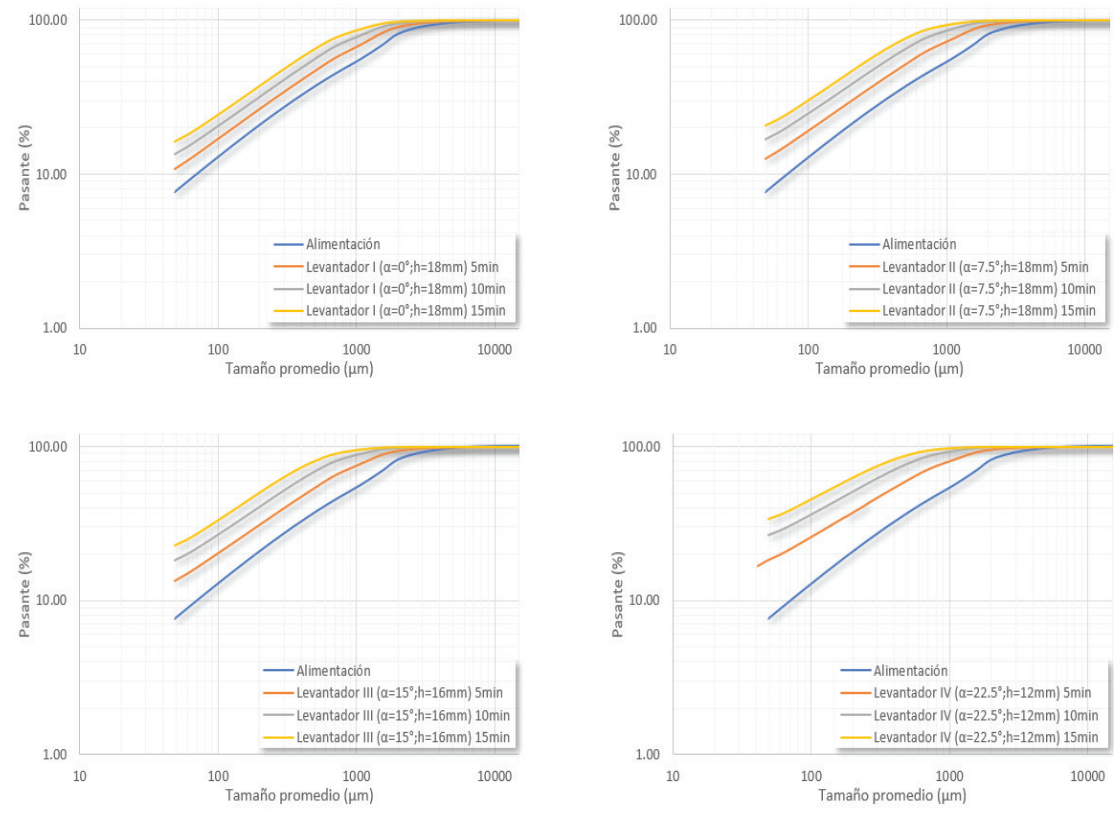

Figura 5. Análisis granulométricos cinéticos con diferente levantador 

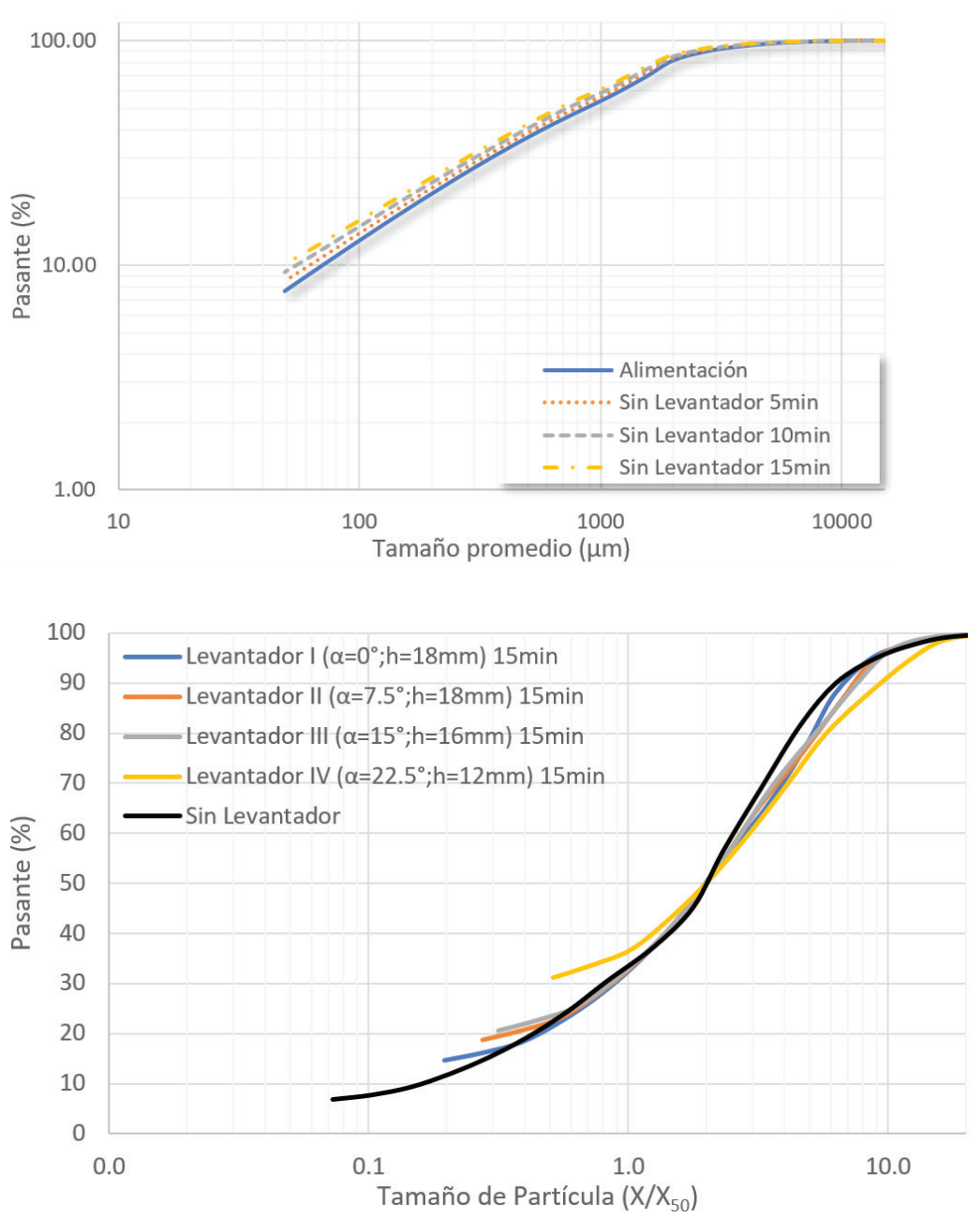

Figura 6. Análisis granulométrico-cinético sin ningún tipo de levantador por lo tanto, denotará la cantidad de finos generados ante el uso de los distintos tipos de levantador.

En la molienda de bolas existen dos tipos principales de mecanismos que dan lugar a la fractura del mineral, el primero de ellos es la fractura por impacto que se da gracias a la corriente de catarata (Figura 1). El mecanismo de impacto genera una distribución de partículas que considera una gran gama de tamaños y es una fractura rápida. Esto se debe a que la energía mecánica que genera el movimiento del cilindro se transforma en energía cinética al trasmitirse por medio de los levantadores a la carga y posteriormente se transforma en energía potencial cuando en el pie de carga sucede la lluvia de impactos. El segundo es el mecanismo de abrasión, el cual se da en la corriente de cascada. Este mecanismo muestra como resultado una gama de tamaños gruesos de los cuales se escienden tamaños de partícula muy finos. Lamentablemente este tipo de mecanismo de fractura es muy lento y consume una gran cantidad de energía. Como se puede apreciar el levantador que tiene la mayor cantidad de ge- neración de partículas finas es el Levantador IV $\left(\alpha=22.5^{\circ} ; \mathrm{h}=12 \mathrm{~mm}\right)$ con casi un $32 \%$ de partículas finas. Es decir, bajo estas consideraciones de operación dadas, es capaz de mantener un equilibrio adecuado entre los dos tipos de mecanismos. El Levantador III ( $\alpha=$ $15^{\circ} ; \mathrm{h}=16 \mathrm{~mm}$ ) es el que tiene el segundo mejor rendimiento, ya que genera un $21.5 \%$ de tamaños finos. Por otro lado, el Levantador II $\left(\alpha=7.5^{\circ} ; \mathrm{h}=18 \mathrm{~mm}\right)$ y el Levantador I ( $\left.\alpha=0^{\circ} ; \mathrm{h}=18 \mathrm{~mm}\right)$ presentan los peores rendimientos con un $14 \%$ y $19 \%$ de finos generados, respectivamente, esto puede explicarse debido a que se favorece solo un tipo de mecanismo, el de impacto.

El diseño de los levantadores estudiados presenta un incremento gradual en su ángulo, para ello fue necesario disminuir la altura de cada uno de los levantadores. Como se puede apreciar, de acuerdo con los datos obtenidos, el factor "ángulo" es de suma importancia y debe tomarse en cuenta para el diseño de los levantadores, debido a que claramente bajo las condiciones de molienda propuestas, hay una relación directamente 
proporcional entre los finos generados y el ángulo del levantador.

\section{RelaCión CONSUMO de energía ReduCCión de tAMAÑo}

La Figura 8 muestra la relación entre el consumo de energía y la cantidad de finos generados. Como se puede apreciar, someter un mineral a un proceso de molienda sin el uso de los levantadores generará una cantidad de finos prácticamente despreciable. Esto se debe a que sin levantadores disponibles la energía mecánica no puede ser suministrada de una manera óptima a la carga. Considerando que la máxima energía consumida de $9.69 \mathrm{kWh} /$ ton; $6.89 \mathrm{kWh} /$ ton que se consumen sin el uso de levantador, representan $71.16 \%$ de la energía total consumida. La ineficiencia de este tipo de molienda es notable ya que solo se generaron $6.76 \%$ de finos, con la energía suministrada. Se puede concluir a partir de los datos obtenidos, que el uso de levantador maximiza los efectos de la molienda, generando las corrientes de catarata y cascada que permite crear un perfil de carga donde los mecanismos de molienda logren la reducción de tamaño.

A partir de los datos obtenidos en la Figura 8 se concluye que el ángulo del levantador tiene efecto sobre el cómo se desarrolla el perfil de carga. El diseño experimental contempla una altura $\mathrm{h}=18 \mathrm{~mm}$ para el tipo de levantadores I y II, sin embargo, el diseño contempla la variación del ángulo entre los Levantadores I y II, que es de $\alpha=0^{\circ}$ para el Levantador I y $\alpha=7.5^{\circ}$ para el Levantador II. Comparando estos dos levantadores, se puede concluir que el Levantador II $\left(\alpha=7.5^{\circ} ; \mathrm{h}=18 \mathrm{~mm}\right)$ con menor cantidad de energía $(9.10 \mathrm{kWh} /$ ton $)$ produce una mayor cantidad de finos $(18.77 \%)$ que el Levantador I ( $\alpha=0^{\circ} ; \mathrm{h}=18 \mathrm{~mm}$ ) el cual tiene un consumo de energía mayor $(9.41 \mathrm{kWh} /$ ton $)$ y una generación de finos menor $(11.86 \%)$.

El Levantador IV $\left(\alpha=22.5^{\circ} ; \mathrm{h}=12 \mathrm{~mm}\right)$ denota la mejor eficiencia de molienda de todas aquellas donde su usaron levantadores, debido a que con $9.69 \mathrm{kWh} / \mathrm{ton}$ produce la mayor cantidad de finos (32\%) mostrada en este estudio. La relación de finos creados por cada $\mathrm{kWh} / \mathrm{ton}$ suministrada es la más alta en todas las pruebas con 3.21. El Levantador III $\left(\alpha=15^{\circ} ; \mathrm{h}=16 \mathrm{~mm}\right)$ es el que tiene un segundo mejor rendimiento con $21.5 \%$ de finos producidos con $9.56 \mathrm{kWh} /$ ton, es decir, una producción de finos de 2.16 por cada $\mathrm{kWh} / \mathrm{ton}$. A partir de este análisis se observa que tanto la altura como el ángulo son factores por considerar en el diseño de los levantadores, ya que ambos tienen una influencia en la distribución de las corrientes de catarata y cascada que dan como resultado una molienda efectiva. A partir de esto se puede denotar que a medida que se incrementa el ángulo de levantador la generación de finos se incrementa, esto debido a que como se aprecia en la Figura 8 por cada $\mathrm{kWh} /$ ton suministrada al molino la cantidad de finos generados se incrementa.

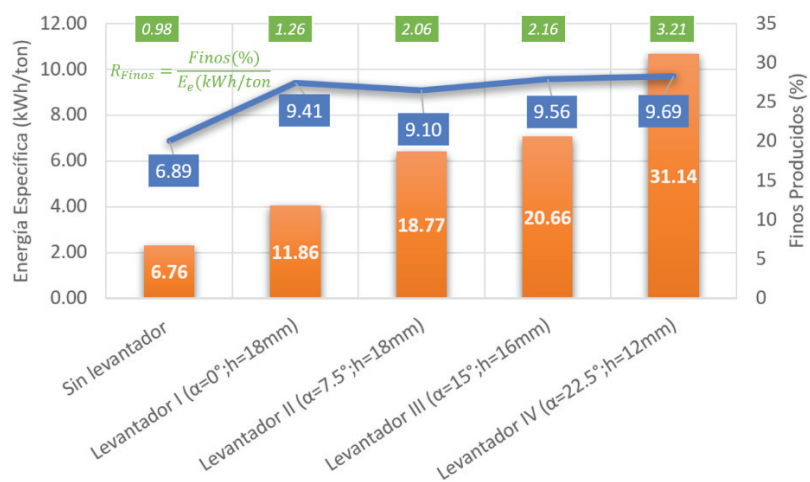

Figura 8. Relación entre porcentaje de finos producidos y consumo de energía específica (kWh/ton) por prueba

\section{Conclusiones}

En este trabajo, se utiliza metodología experimental para analizar los efectos de diseño a considerar en la fabricación de levantadores para molinos rotatorios de bolas. Las condiciones (diseño y operación) y la distribución del tamaño de la alimentación se mantuvieron constantes. La misma velocidad crítica, la cantidad de carga de la bola y mineral fueron usados. El enfoque cinético se utilizó para calcular los efectos del tipo de levantador para maximizar la generacion finos en la molienda. Las siguientes conclusiones se extrajeron del estudio:

- La muestra sometida a molienda sin el uso de un levantador tiene un consumo de energía de $6.89 \mathrm{kWh} / \mathrm{ton}$ es el consumo más bajo registrado. La prueba que tiene el menor consumo de energía cuando se usa un levantador es la que corresponde al Levantador II cuyo ángulo $\alpha=7.5^{\circ}$.

- De acuerdo con los resultados mostrados con uso y no usando levantador se pueda inferir que los levantadores magnifican la molienda, ya que estos generan las corrientes de catarata y cascada que dan origen a los mecanismos de molienda de impacto y abrasión. El análisis de la Figura 6 demuestra sin ningún levantador que la molienda se da por el mecanismo de abrasión, el cual genera fractura lenta.

- El ángulo del levantador tiene efecto sobre el cómo se desarrolla el perfil de carga, ya que la única variación entre el Levantador I $\left(\alpha=0^{\circ} ; \mathrm{h}=18 \mathrm{~mm}\right)$ y el Levantador II $\left(\alpha=7.5^{\circ} ; \mathrm{h}=18 \mathrm{~mm}\right)$, es la variación 
del ángulo. Como se ha discutido con anterioridad la cantidad de finos producidos es mayor en el Levantador II, el cual tiene esta variación en el ángulo.

- El Levantador IV $\left(\alpha=22.5^{\circ} ; \mathrm{h}=12 \mathrm{~mm}\right)$ denota la mejor eficiencia de molienda, con $9.69 \mathrm{kWh} /$ ton produce la mayor cantidad de finos (32\%) mostrada en este estudio. La cantidad de finos creada por cada $\mathrm{kWh} /$ ton es de 3.21. El Levantador III $\left(\alpha=15^{\circ} ; \mathrm{h}=16 \mathrm{~mm}\right)$ es el que tiene un segundo mejor rendimiento con $21.5 \%$ de finos producidos con $9.56 \mathrm{kWh} / \mathrm{ton}$. Al hacer un comparativo entre ambos se concluye que tanto la altura como el ángulo son factores por considerar en el diseño de los levantadores, ya que ambos tienen una influencia en la distribución de las corrientes de catarata y cascada que dan como resultado una molienda efectiva.

- Los autores recomiendan considerar los parámetros de ángulo y altura en los futuros diseños de levantadores, así mismo, realizar variaciones en las condiciones operacionales de la molienda para entender del todo los efectos de los parámetros mencionados.

\section{Agradecimientos}

Los autores agradecen a SEP por el apoyo financiero a través del programa de reconocimiento al perfil deseable No. 511-6/2019-9705.

\section{Referencias}

Bond, F.C. (1961). Crushing and grinding calculations. Brit. Chem. Eng. Part I 6 (6), 378-385 Part II 6 (8) 543-548.

Bulled D, Husain K. \& Lozano C. (2008). Small scale test to determine work index for high pressure grinding rolls. PROCEMIN, Santiago, Chile, 22-24 de octubre.
Bhattacharyya, A., Tuzcu, E.T. \& Rajamani, R. (2016). Experimental study on non-linear behavior of breakage rates due to fines generation in wet batch milling. Minerals Engineering, 99, 1929. http://dx.doi/10.1016/j.mineng.2016.09.018

Cleary, P.W. (1998). Predicting charge motion, power drawn, segregation, wear and particle breakage in ball mills using discrete element methods. Miner. Eng. 11, 1061-1080.

Colman, R. (1983). Metallurgical testing procedures. Mineral Processing Plant Design. 2a ed. AIME (pp. 144-182, Chapter 9).

Geng, F., Gang, L., Wang, Y., Li, Y. \& Yuan, Z. (2015). Numerical investigation on particle mixing in a ball mill. Powder Technol., 292, 64-73.

Morrison, R.D. \& Cleary, P.W. (2008). Towards a virtual comminution machine. Minerals Engineering Journal, 21. 770-781.

Djordjevic, N. (2005). Influence of charge size distribution on netpower daw of tumbling mill based on DEM modelling. Miner. Eng., 18, 375-378

Moys, M.H., Van Nierop, M.A. \& Smit, I. (1996). Progress in measuring and modeling load behavior in pilot and industrial mills. Miner. Eng., 9 (12), 1201-1214.

Powell, M.S. \& McBride, A.T. (2006). What is required from DEM simulation to model breakage in mills? Miner. Eng., 19, 10131021.

Powell, M.S. \& McBride, A.T. (2004). A three-dimensional analysis of media motion and grinding regions in mills. Miner. Eng., 17, 1099-1109.

Hosten C. \& Cimilli H. (2009). The effects of feed size distribution on confined-bed comminution of quartz and calcite in pistondie press. Int. J. Miner. Process., 91, 81

Rajamani, R.K. \& Mishra, B.K. (1996). Dynamics of ball and rock charge in sag mills. In Proc. SAG (Vol. 19).

Rosales-Marín, G., Delgadillo, J.A., Tuzcu, E.T. \& Pérez-Alonso, C.A. (2016) Prediction of a piston-die press product using batch population balance model. Asia-Pac. J. Chem. Eng., 11: 1035-1050. http://dx.doi/10.1002/apj.2044. 